\title{
Non-uniform Deformation Behavior of Coarse-grained Ultralow Carbon Steel Measured Using Digital Image Correlation Method
}

\author{
Takayuki HAMA, ${ }^{1) *}$ Takuna NISHI, ${ }^{1)}$ Masashi OKA, ${ }^{1)}$ Takashi MATSUNO, ${ }^{2)}$ Yoshitaka OKITSU, ${ }^{3)}$ Seiji HAYASHI, ${ }^{3)}$ \\ Kenji TAKADA ${ }^{3)}$ and Hirohiko TAKUDA ${ }^{1)}$ \\ 1) Graduate School of Energy Science, Kyoto University, Yoshida-Honmachi, Sakyo-ku, Kyoto, 606-8501 Japan. \\ 2) Tottori University, 4-101 Koyama-cho-minami, Tottori, Tottori, 680-8552 Japan. \\ 3) Honda Motor Co., Ltd. 4630 Shimotakanezawa, Haga-machi, Haga-gun, Tochigi, 321-3393 Japan.
}

(Received on November 18, 2020; accepted on February 9, 2021)

\begin{abstract}
In this work, non-uniform deformation behavior of coarse-grained ultralow carbon steel was investigated under uniaxial tension and cyclic simple shear. A digital image correlation method was used to measure strain fields at the grain level. Heterogeneous strain distribution appeared at the early stage of the process, and the heterogeneity remained almost unchanged in the subsequent deformation. Localized strain bands were observed under both uniaxial tension and simple shear, but the directions of the bands were different between the two deformation modes. It was hypothesized that the directions of the strain bands were correlated with the planes where the maximum or minimum shear stresses occurred. During cyclic simple shear, the heterogeneity of strain distribution and occurrence of strain bands depended on the shear direction. In contrast, the number of cycles had little effect on the heterogeneity of strain distribution. Comparing the grain boundaries and the strain distributions, it was observed that many of the strain-band boundaries occurred along the grain boundaries, but some of them appeared within grains.
\end{abstract}

KEYWORDS: ultralow carbon steel; oligocrystal; digital image correlation; simple shear test; cyclic loading.

\section{Introduction}

Macroscopic deformation behavior in polycrystalline metals depends on microscopic deformation at the grain level that occurs under macroscopic boundary conditions. It is understood that microscopic deformation at the grain scale is affected by various factors, such as the anisotropic deformation of the crystalline slip, the size and shape of grains, the crystal orientations, and the misorientations between neighboring grains. ${ }^{1,2)}$ Accordingly, the microscopic deformation is usually non-uniform even when macroscopically uniform deformation and boundary conditions are given to metals. Microscopic non-uniform strains usually yield localized deformation, which in some cases results in fracture. For example, Nakada et al. ${ }^{3)}$ studied heterogeneous strain distribution in a dual-phase steel and reported that large strain bands formed in the ferrite phase lead to ductile fracture in the martensite phase. Hasegawa et $a l .{ }^{4)}$ reported that the propagation of microcracks is different depending on the stretch-flange-formability of dual-phase steels. Therefore, to understand comprehensively the plastic deformation behavior of polycrystalline metallic materials,

\footnotetext{
* Corresponding author: E-mail: hama@energy.kyoto-u.ac.jp
}

it is important to investigate the microscopic non-uniform deformation behavior at the grain scale.

Many studies have been conducted to investigate the microscopic deformation, specifically strain fields, at the grain scale of polycrystalline metals. ${ }^{1-3,5-20)}$ An abundant literature survey was given by Efstathiou et al. ${ }^{7)}$ An accurate measurement of strain fields at the grain scale is important to understand the deformation characteristics. Among several techniques for strain measurements used for this purpose, ${ }^{5)}$ digital image correlation (DIC) methods have often been used to study quantitatively strain distributions at the grain scale. ${ }^{1-3,7-20)}$ DIC methods allow quantitative evaluations of strain fields on the basis of random speckle patterns given prior to the tests and digital images captured during deformation. Abuzaid et al. ${ }^{8)}$ conducted a uniaxial tensile test of a nickel-based superalloy, Hastelloy X, under an optical microscope and measured the strain distribution using a DIC method. They also estimated the magnitude of the residual Burgers vectors and plastic strains due to slip propagation across grain interfaces by comparing the obtained strain distributions with the crystal orientation distributions. Guo et al. ${ }^{9)}$ investigated tensile deformation behavior of a copper bicrystal using a DIC method and scanning electron microscopy (SEM). They reported that 
the strains near grain boundaries are significantly smaller than those within the two grains. Sachtleber et al. ${ }^{2)}$ pointed out that strain localization tended to initiate at grain boundary triple junctions, and strain bands appeared along grain boundaries inclined $45 \mathrm{deg}$ to the loading direction. Similar results were also reported by Efstathiou et al. ${ }^{7)}$ and Tabata et al. $^{21)}$ Tabata et $a .^{21)}$ performed uniaxial tensile tests of ferrite alloys in SEM and investigated the strain evolution during the deformation using a DIC method. They reported that in the initial stage of deformation, plastic strains occur locally near the triple and quadruple junctions of grain boundaries, and they tend to be connected with each other in the diagonal direction to the tensile direction.

Because grain sizes of metals used for structural components are usually several tens of microns, most of the aforementioned studies were conducted by using microscopy. Although the combination of DIC methods and microscopy is effective and can provide a lot of information on the relationship between macroscopic and microscopic deformation behaviors, there are some drawbacks. The two major issues are explained below. First, the deformation modes that can be given to the specimens are quite limited. Because materials are often subjected to various deformations in the industrial forming processes, including tension, compression, shear, and cyclic loadings, it is significant to study the plastic deformation behavior under different deformation modes. However, most of the previous studies ${ }^{1,3,6-20)}$ have been limited to uniaxial tensile tests presumably because of the limitation of experimental setups; thus, the effects of the deformation modes on microscopic and macroscopic deformation behaviors are not yet understood.

Second, it is not trivial to give fine speckle patterns to observe microscopic strain distributions at the grain scale for metals with grain size of several tens of microns. Black and white color sprays are usually used to give speckle patterns for DIC measurements. Because this procedure is not available to observe strain fields within grains with grain sizes of several tens of microns, a lot of efforts have been done to give fine speckle patterns at the grain scale, including etching ${ }^{11,14,21)}$ and deposition of nanoparticles, such as colloidal silica particles ${ }^{13)}$ and gold nanoparticles. ${ }^{12,22)}$ These methods are clearly more difficult to handle compared to the conventional procedure using sprays.

To address the aforementioned technical drawbacks, it would be useful to utilize coarse-grained specimens. By coarsening the crystal grains, it is expected that the strain distributions and their evolution can be measured with sufficient resolution even with speckle patterns given by the conventional procedure. In addition, because the strain fields at the grain scale can be measured without using microscopy, the measurement of strain fields at the grain scale can be conducted under various deformation modes, including simple shear and cyclic loading, using existing experimental apparatuses. Thus, the effects of the deformation modes on the microscopic deformation behavior can be investigated under fine spatial and time resolutions.

Coarse-grained materials have been used to investigate microscopic deformation at the grain scale. ${ }^{1,2,9,22-29)}$ Zhang and Tong ${ }^{25}$ conducted a uniaxial tensile test using a coarse-grained $\mathrm{Al}-0.5 \% \mathrm{Mg}$ specimen, and investigated microscopic heterogeneous deformation, including strain distributions and active slip systems observed by slip plane trace observations. They reported that the crystals do not obey the Taylor model. Zhao et al. ${ }^{26)}$ studied strain localization, surface roughening, microstructure, and in-grain fragmentation of a coarse-grained pure Al under uniaxial tension by experiments and crystal-plasticity finite-element simulations. They reported that the absence of grain boundaries as dislocation barriers results in easy occurrence of strain localization. It was also found that surface roughening correlated with strain localization. Lim et al. ${ }^{27)}$ examined the microscopic deformation behavior of a coarse-grained tantalum sheet under uniaxial tension by means of experiments and a crystal-plasticity finite-element method. The simulation results were in good agreements with experimental results. In addition, numerical experiments suggested that the choice of $\{110\}$ or $\{112\}$ slip systems had little effect on the simulation results. Baudoin et al. ${ }^{28)}$ investigated strain evolution during uniaxial tension of a coarse-grained commercially-pure titanium sheet using a DIC method and a crystal-plasticity finite-element method. The strain distribution, its evolution and the slip lines observed in the experiments were reproduced qualitatively well in the simulations. Furthermore, the effect of critical resolved shear stress of the basal slip system on the predictive accuracy was also investigated. However, the past studies have focused their attentions on uniaxial tensile deformation, and studies on other deformation modes are scarce.

In this study, experiments of tensile and simple shear tests were conducted on a coarse-grained ultralow carbon steel sheet. The microscopic strain distribution and its evolution, and the effect of the deformation mode on the strain distributions were examined in detail. Moreover, cyclic simple shear tests were also conducted, and the effect of cyclic deformation on the strain distributions was also investigated.

\section{Experimental Procedure}

\subsection{Material}

An ultralow carbon steel with coarse grains used in this study was prepared as follows. A slab of ultralow carbon steel was annealed at $1250^{\circ} \mathrm{C}$ for $2 \mathrm{~h}$ in nitrogen atmosphere, followed by furnace cooling. Then, the slab was reheated and annealed at $700^{\circ} \mathrm{C}$ for $1 \mathrm{~h}$ in nitrogen atmosphere, followed by furnace cooling. The sheet specimens for tensile and simple shear tests were cut from the coarsegrained slab. The chemical composition is shown in Table 1. Figure 1(a) shows the sample geometry for the uniaxial tensile test. The dumbbell-shaped specimen had a gauge size of $15.0 \mathrm{~mm} \times 7.1 \mathrm{~mm}$. The thickness was set to 0.4 or 0.6 $\mathrm{mm}$. Figure 1(b) shows the geometry for the simple shear test. The rectangular specimen had a gauge size of 17.0 $\mathrm{mm} \times 3.0 \mathrm{~mm}$. The notches with the curvature radius of $1.5 \mathrm{~mm}$ were provided to give uniform deformation in the gauge area. The thickness was set to $0.4 \mathrm{~mm}$.

Figure 2 shows the inverse pole figure map measured

Table 1. Chemical composition of material (mass\%).

\begin{tabular}{cccccccc}
\hline $\mathrm{C}$ & $\mathrm{Si}$ & $\mathrm{Mn}$ & $\mathrm{P}$ & $\mathrm{S}$ & $\mathrm{Ti}$ & Sol-Al & $\mathrm{N}$ \\
\hline 0.0020 & 0.01 & 0.07 & 0.015 & 0.007 & 0.038 & 0.029 & 0.0017 \\
\hline
\end{tabular}


(a)

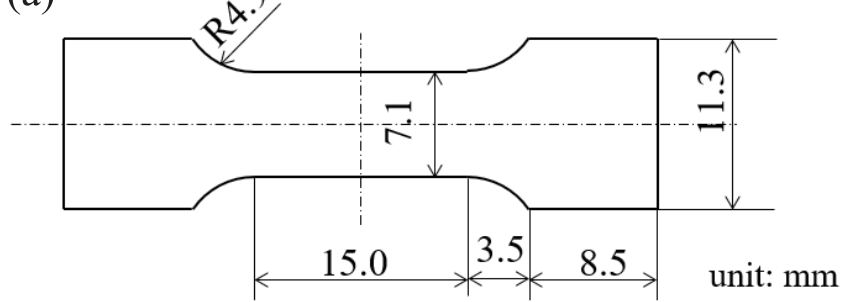

15

(b)

(i)

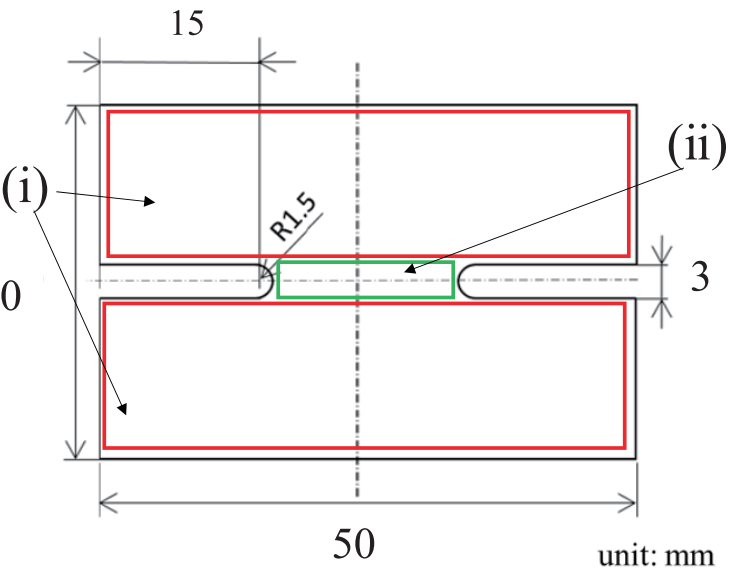

Fig. 1. Geometries of specimens for (a) uniaxial tensile and (b) simple shear tests. (Online version in color.)

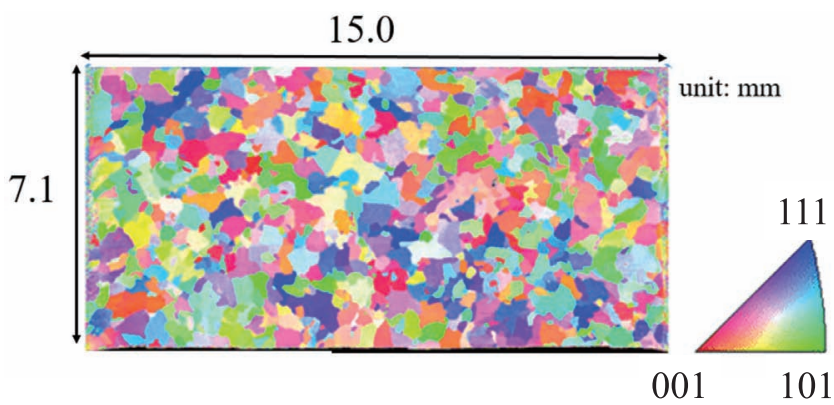

Fig. 2. Inverse pole figure map of initial material. (Online version in color.)

on an initial specimen, obtained from the Electron Backscattered Diffraction (EBSD) measurement. The crystal orientations along the normal direction of the specimen are shown. The specimen had a ferrite single-phase structure with nearly-random crystal orientations. The average grain size was approximately $0.47 \mathrm{~mm}$.

\subsection{Uniaxial Tensile Test}

To utilize a DIC method, speckle patterns with black and white color sprays were painted on the surface of the gauge section by using an airbrush. The painted surface was air dried for one day before the experiment was conducted. The uniaxial tensile test was conducted at the initial strain rate of $6.7 \times 10^{-3} \mathrm{~s}^{-1}$. The specimen was stretched until the average tensile strain reached 0.05. A single monochrome CMOS camera (Point Gray Research, Inc. Grasshopper3 GS3-U351S5) and a bi-telecentric lens (Opto Engineering TC23024) were used to capture images of the speckle patterns during the test. The sampling frequency was $1 \mathrm{~Hz}$. Then, a twodimensional DIC analysis was performed to observe inplane strain distributions on the gauge section. The GOM

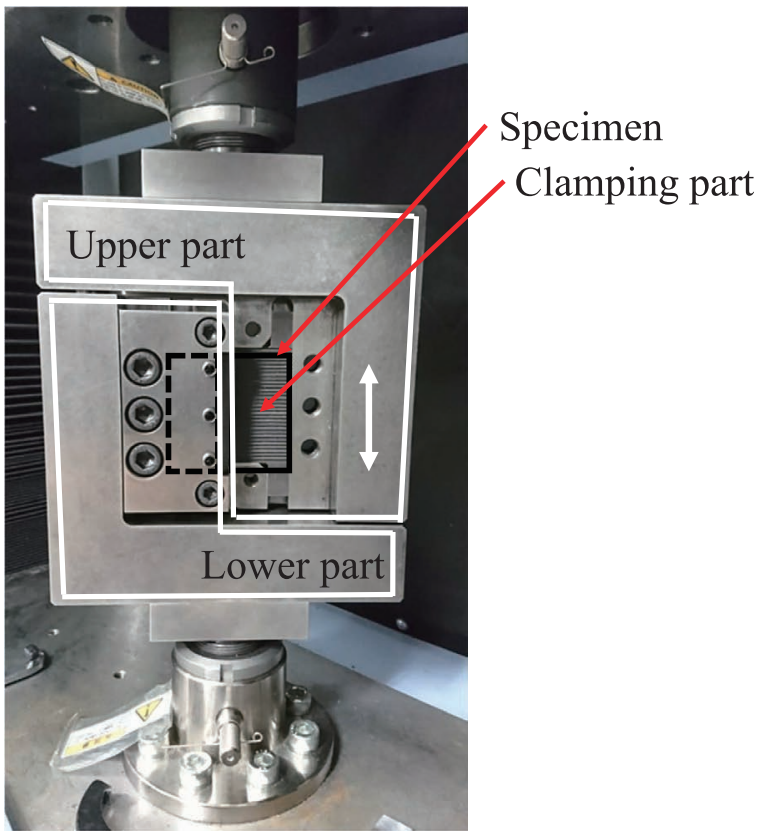

Fig. 3. Photograph of simple shear testing device. (Online version in color.)

Correlate Professional V8 software (GOM mbH) was used for the analyses. The number of pixels was $2448 \times 2048$, and the physical size of a pixel was approximately $3.45 \mu \mathrm{m}$. A subset size was $21 \times 21$ pixel.

In addition, a three-dimensional DIC system ARAMIS (GOM $\mathrm{mbH}$ ) with two cameras was used to investigate in-plane strain distribution and out-of-plane surface roughness evolution. The ARAMIS Professional software (GOM $\mathrm{mbH}$ ) was used for the analyses. The number of pixels of the two cameras for the three-dimensional analysis was $2752 \times 2200$, and the physical size of a pixel was approximately $11.4 \mu \mathrm{m}$. A subset size was $21 \times 21$ pixel.

\subsection{Simple Shear Test}

Speckle patterns were painted on the specimen as in the case of the tensile test. The simple shear testing device used in this study is shown in Fig. 3. First, the grippers of the shear specimen (Fig. 1(b), area (i)) were set in the clamping part of the device, and fixed using bolts so that deformation in the width direction was restrained. Then, while fixing the lower part, the upper part of the device was moved to give simple shear deformation to the gauge section (Fig. 1(b), area (ii)). The initial strain rate was set to $2.5 \times 10^{-3} \mathrm{~s}^{-1}$. The two-dimensional DIC analysis was performed using the same system and conditions as those of the tensile test.

One of the features of the simple shear test is that cyclic loading tests can be conducted without involving out-ofplane buckling. ${ }^{30)}$ Therefore, in the present study, the specimens were subjected to cyclic loadings with the amplitude of average mathematical shear strain of approximately 0.1 for three cycles.

\section{Results and Discussion}

\subsection{Uniaxial Tensile Test}

3.1.1. Strain Distribution and its Evolution

Figure 4 shows the nominal stress-average equivalent 
strain curve obtained from the uniaxial tensile test using the $0.4 \mathrm{~mm}$ thick specimen. The horizontal axis is the equivalent strain averaged over the entire gauge section. To use the same strain measure in the uniaxial tensile and simple shear tests, a von Mises equivalent strain was used in this paper. It should be noted that the total strains, referred to the undeformed configuration, were calculated assuming the incompressibility and were used to calculate the von Mises equivalent strain.

The equivalent strain distributions in the gauge section with the size of $7.1 \mathrm{~mm} \times 15 \mathrm{~mm}$ at the average equivalent strains of $0.004,0.012,0.02,0.03$, and 0.04 , which correspond to the points 1, 2, 3, 4 and 5 in Fig. 4, respectively, are shown in Fig. 5. Strain ranges are the same regardless of the result. As the average equivalent strain increased, band-shaped localized strains became gradually pronounced in the diagonal direction. Similar results were also reported in literatures. ${ }^{2,7,21)}$ To investigate the evolution of the strain localization in detail, the strain ranges of the figures were adjusted depending on the average equivalent strain, see Fig. 6. Apparently, the strain localization already started to be formed at the average equivalent strain of 0.004 (Fig. 6(a)), and in the following deformation processes the magnitude of the localized strains gradually increased but the positions of the localized strains remained almost unchanged. This result suggests that the locations where strain localization occurred were determined in the early stage of deformation.

To investigate the evolution of the non-uniform strain distribution quantitatively, the evolution of equivalent strains measured at the points A-H shown in Fig. 6(a) as a function of the average equivalent strain is shown in Fig. 7. The points A-D were selected from the strain-localized areas, whereas the points $\mathrm{E}-\mathrm{H}$ were taken from the areas with relatively small strains. The equivalent strains at the points $\mathrm{A}-\mathrm{D}$ were larger than those at the points $\mathrm{E}-\mathrm{H}$ throughout the process. This result is consistent with the abovementioned discussion that the locations of the strain localization were determined in the early stage of the deformation. Interestingly, the strains increased almost linearly irrespective of the point. These results suggest that, in the strain range tested in this study, the strain evolution at each

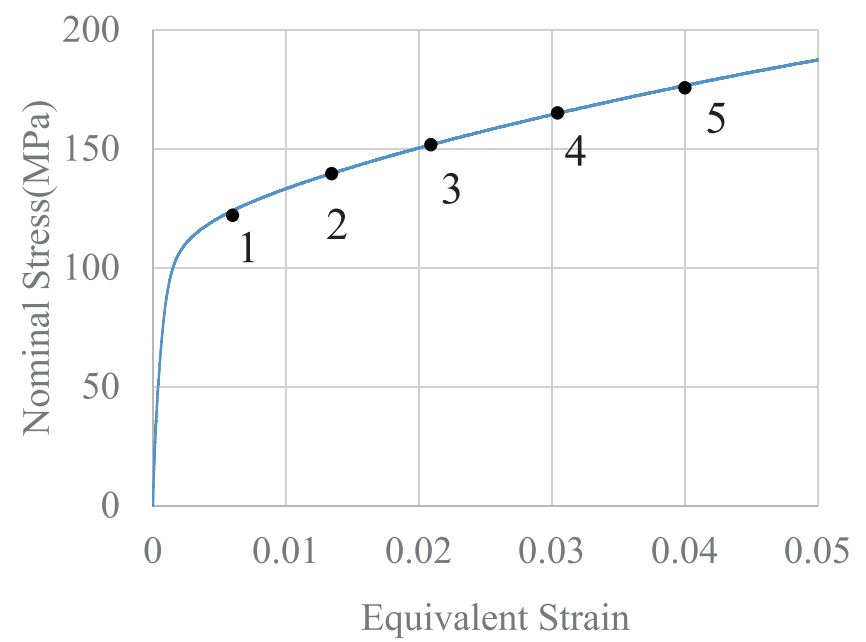

Fig. 4. Nominal stress-average equivalent strain curve under uniaxial tension. (Online version in color.) point was rather uniform and did not change notably during the deformation although the overall strain distribution in the gauge section was non-uniform.

As explained earlier, band-shaped localized strains appeared in the diagonal direction of the tensile direction. In this study, a localized strain band with the magnitude larger than $125 \%$ of the average equivalent strain is termed as the strain band. The angles of the strain bands to the tensile direction was estimated as follows. First, 16 strain bands were selected arbitrarily from the strain distribution at the average equivalent strain of 0.04 (Fig. 6(d)). Then, for simplicity, the bands were approximated by rectangles, and their angles to the tensile direction were measured by using the software Adobe Photoshop. As a result, the angles of 16 bands were in the range from $40.5^{\circ}$ to $73.9^{\circ}$, consistent with previous studies. ${ }^{2,7,21)}$ Because the macroscopic maximum and minimum shear stresses act on the planes inclined $45^{\circ}$ from the tensile direction in the case of uniaxial tension, it

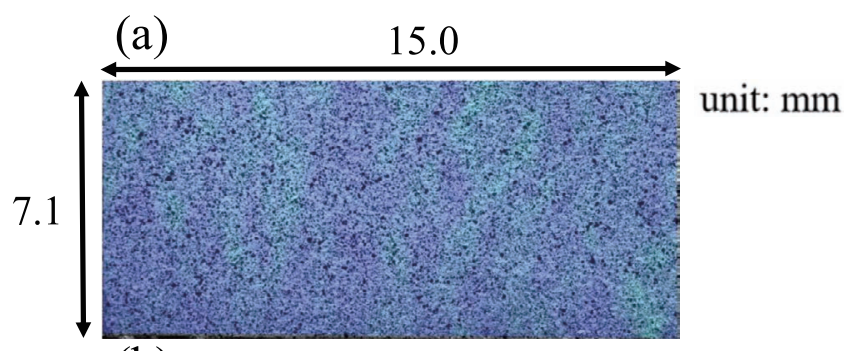

(b)

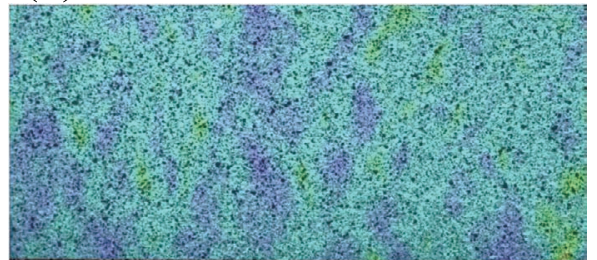

(c)

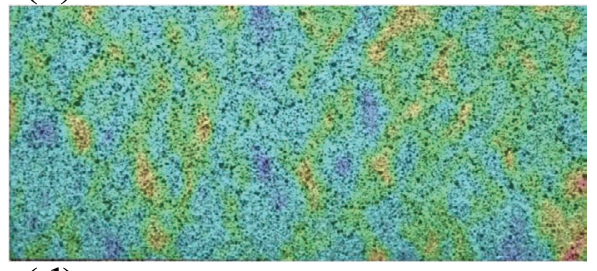

(d)

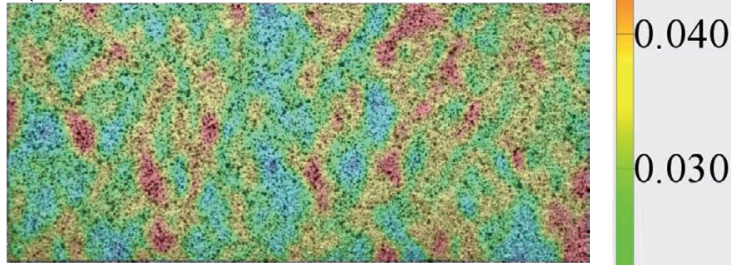

(e)

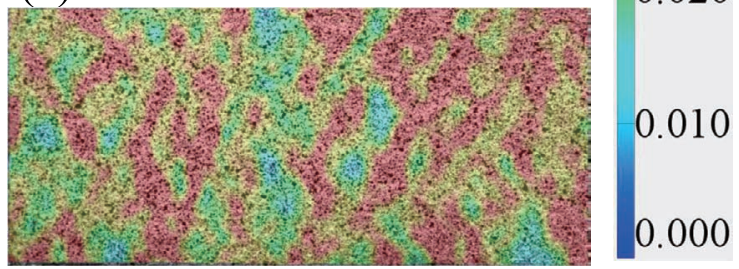

Fig. 5. Equivalent strain distributions under uniaxial tension measured at points (a) 1, (b) 2, (c) 3, (d) 4, and (e) 5 shown in Fig. 4. (Online version in color.) 

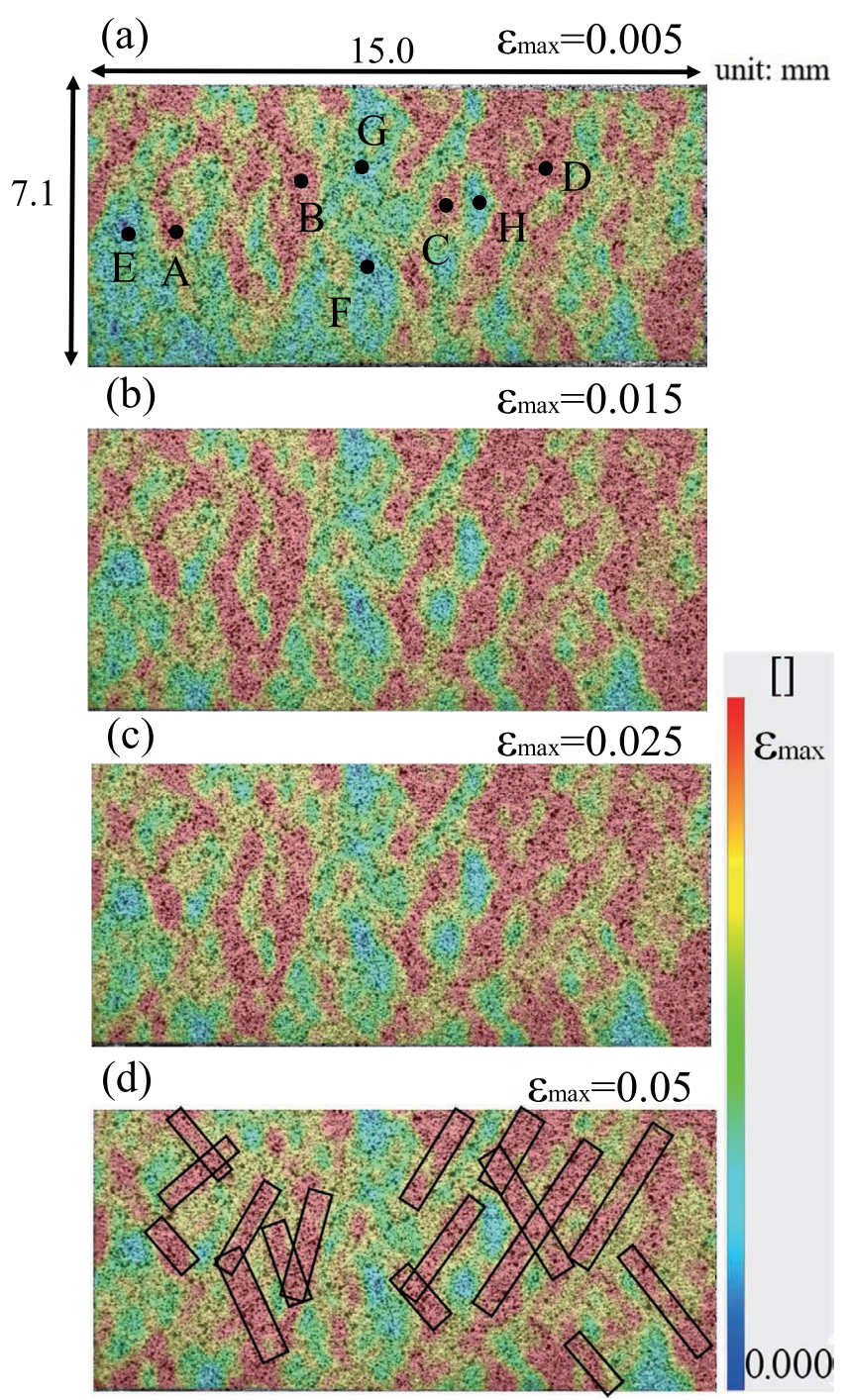

\subsubsection{Relationship between In-plane Strain Distribution} and Out-of-plane Deformation

Because the aforementioned results discussed only the two-dimensional strain evolution, the effect of out-of-plane deformation on the in-plane distribution is not understood. Therefore, the relationship between the evolution of in-plane equivalent strain distribution and out-of-plane deformation was investigated using the results of the three-dimensional DIC analysis. The results are shown in Fig. 8. It should be noted that the specimen used in this test was different from that of Figs. 4-7; thus, the equivalent strain distributions shown in Fig. 8 are different from those of Fig. 5. There is no clear correlation in the distribution patterns between the equivalent strain and the out-of-plane displacement. This result indicates that the development of surface roughness had little effect on the development of in-plane strain distribution and strain bands, suggesting that large in-plane strains did not always involve large out-of-plane displacements.

\subsubsection{Effect of Through-thickness Deformation on Strain Band}

To further study the effect of out-of-deformation, the aforementioned results obtained using the specimen with $0.4 \mathrm{~mm}$ thickness were compared with those of the specimen with $0.6 \mathrm{~mm}$ thickness. Because the average grain size was approximately $0.47 \mathrm{~mm}$, the specimen with $0.4 \mathrm{~mm}$ thickness would have only one or two grains through the thickness at most, whereas the specimen with $0.6 \mathrm{~mm}$ thickness would have multiple grains. Therefore, the throughthickness deformation would be different between the two samples.

Figure 9 shows the equivalent strain distribution at the is likely that these microscopic strain bands developed in the vicinity of the planes where the macroscopic maximum and minimum shear stresses occurred. average equivalent strain of 0.04 for the specimen with 0.6 $\mathrm{mm}$ thickness. The development of strain bands seemed similar to that of the specimen with $0.4 \mathrm{~mm}$ thickness (Fig. 


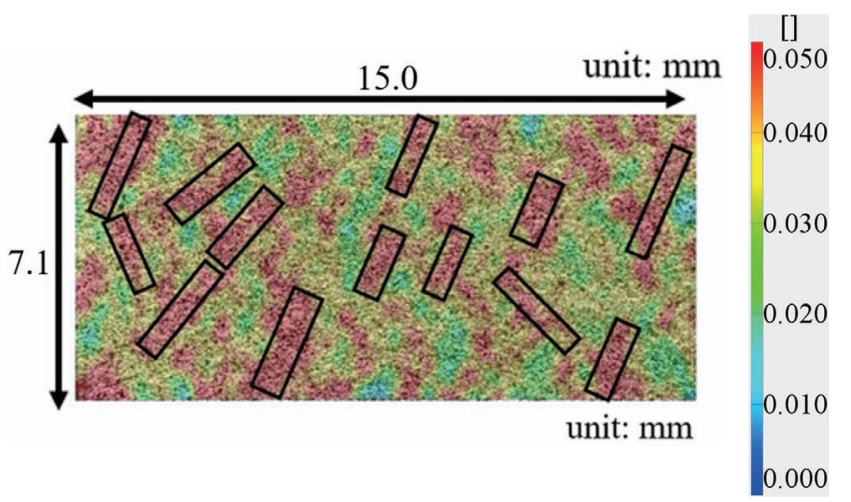

Fig. 9. Equivalent strain distributions under uniaxial tension measured at average equivalent strain of 0.04 obtained for specimen with a thickness of $0.6 \mathrm{~mm}$. (Online version in color.)

6(d)). To examine quantitatively, the width and the angles to the tensile direction of the strain bands were compared between the two specimens. Thirteen arbitrary strain bands were selected and approximated by rectangles as in Fig. 6(d), and the width and angle of each rectangle were measured. The average widths of the strain bands were approximately $0.55 \mathrm{~mm}$ and $0.53 \mathrm{~mm}$ for the $0.4 \mathrm{~mm}$ and $0.6 \mathrm{~mm}$ thickness specimens, respectively. The angles of 13 bands for the $0.6 \mathrm{~mm}$ thickness specimen were in the range from $38.9^{\circ}$ to $67.0^{\circ}$. The differences between the two samples were within the errors of the measurements, suggesting that the difference in the through-thickness deformation had little effect on the development of strain bands. This result supports the result explained in Section 3.1.2.

\subsection{Simple Shear Test}

\subsubsection{Strain Distribution and its Evolution}

Figure 10(a) shows the shear stress-shear strain curve obtained from the cyclic simple shear test. Shear strain was calculated from the stroke of the device. Figure 10(b) shows the evolution of the average equivalent strain in the gauge section calculated from the DIC measurement. It should be noted that, for simplicity, time was used for the horizontal axis and the beginning of each cycle was defined to be time $t=0 \mathrm{~s}$. Moreover, the evolution curve was shown separately for the three cycles for the visibility purposes. For reference, the relationship between the stroke and the time is also shown. In the following, the deformation ranges with positive and negative shear strains are referred to as positive and negative shear, respectively, and they are designated as the regions (i) and (ii), respectively, as shown in Fig. 10. The qualitative trend of average equivalent strain evolution was almost independent of the cycle.

The equivalent strain distributions in the gauge section with the size of $3.0 \mathrm{~mm} \times 17 \mathrm{~mm}$ for the first cycle measured at the points a to f shown in Fig. 10 are shown in Fig. 11. It should be noted that the average equivalent strains in Figs. 11(a) and 11(d), Figs. 11(b) and 11(e), and Figs. 11(c) and 11(f) were the same to each other. Clear strain bands appeared in all the results, as in the case of uniaxial tension. However, unlike uniaxial tension, the strain bands were developed in the perpendicular and horizontal directions of the gauge section. Because the macroscopic maximum and minimum shear stresses acted on the planes in the horizontal (a)
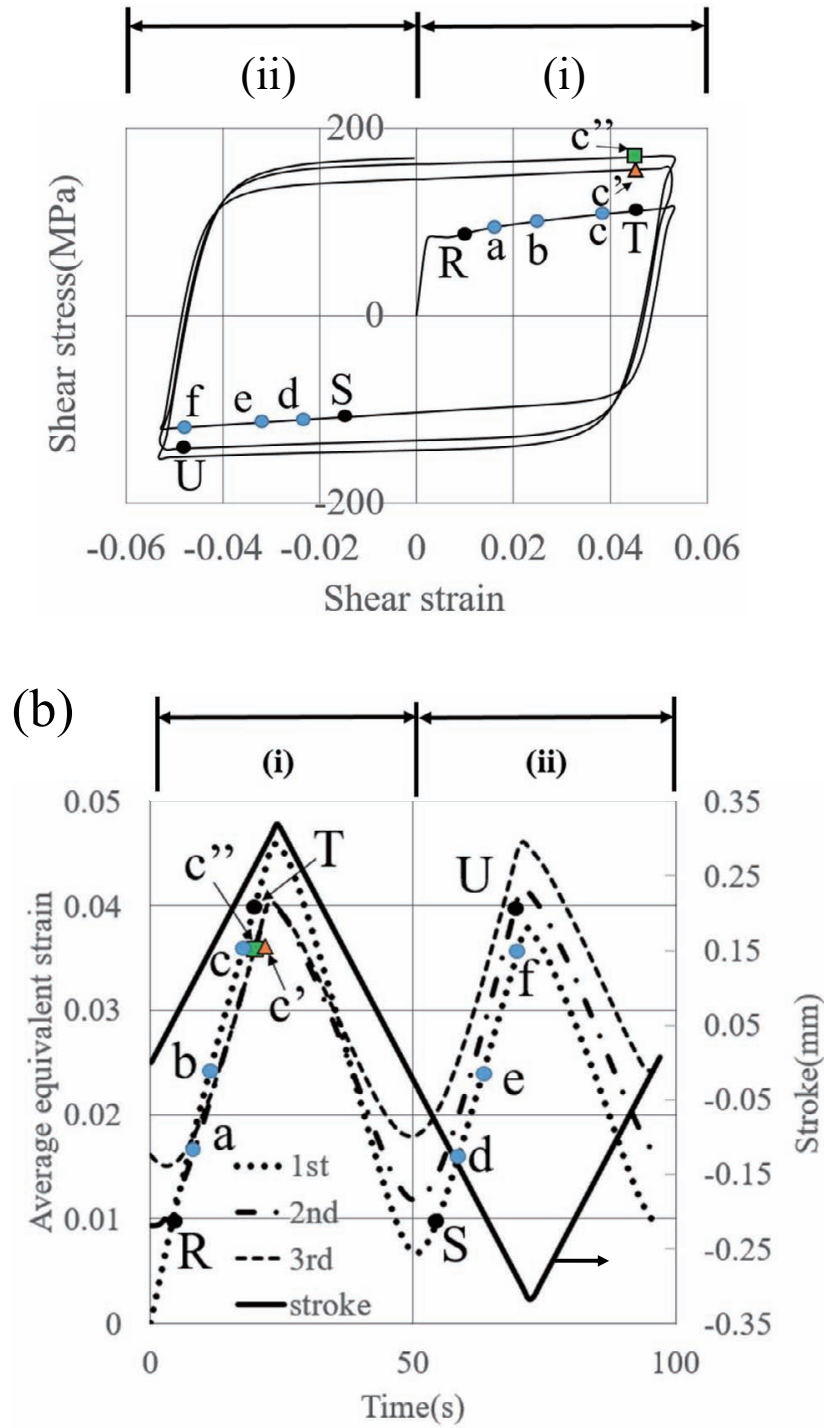

Fig. 10. Evolution of strains under cyclic simple shear. (a) Average shear stress-average shear strain curve, and (b) evolution of average equivalent strain as a function of time for each cycle. (Online version in color.)

and vertical direction of shear, it is likely that these strain bands developed in the vicinity of the planes where the macroscopic maximum and minimum shear stresses occurred, which confirms the hypothesis presented in Section 3.1.1. The reason that microscopic strain bands are governed by macroscopic stress states will be studied in our future work.

As explained earlier, the strain bands started to be formed at the point a (Fig. 10), as shown in Fig. 11(a), and the positions of the strain localization remained almost unchanged during the positive shear, see Figs. 11(b) and 11(c). This result is consistent with the result of uniaxial tension, suggesting that this tendency is independent of the deformation mode. A similar tendency was observed during the negative shear, see Figs. 11(d), 11(e), and 11(f). However, the strain distribution is apparently different between the positive and negative shear, indicating that the pattern of strain localization depended on the shear direction.

Therefore, the difference in the strain distributions between the positive and negative shear was investigated in 


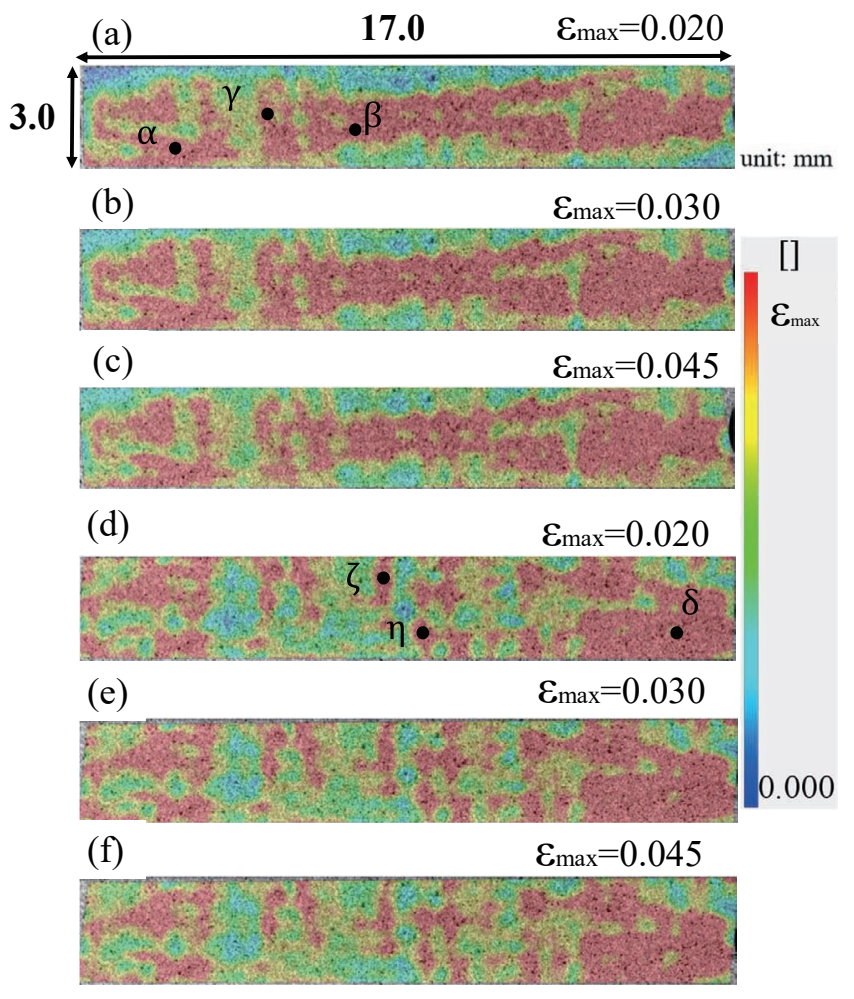

Fig. 11. Equivalent strain distributions under cyclic simple shear measured at points (a) a, (b) b, (c) c, (d) d, (e) e, and (f) f shown in Fig. 10. (Online version in color.)

detail. The local strain evolution during the first cycle was measured at the points $\alpha-\eta$ depicted in Figs. 11(a) and 11(d), and is shown in Fig. 12. The points $\alpha, \beta$ and $\gamma$ were selected from the strain localized areas during the positive shear, whereas the points $\delta, \zeta$ and $\eta$ were taken from the localized area during the negative shear. The equivalent strains increased or decreased almost linearly with the stroke of the device, irrespective of the point. However, the slopes of the strain evolution and the timing of the transition from the positive to negative shear differed depending on the point. These differences would have resulted in the different strain distributions between the positive and negative shear. These findings indicate that, although the macroscopic stress-strain curves were similar between the positive and negative shear as shown in Fig. 10(a), the microscopic strain fields were not necessarily the same but had directional dependences.

Next, the effect of cyclic loading on the strain distribution was investigated. Figures 13(a), 13(b) and 13(c) show the strain distributions measured at the average equivalent strain of approximately 0.036 for the first, second and third cycles, i.e., the points c, c' and c' in Fig. 10, respectively. The strain distributions were very similar irrespective of the number of cycles. Although detailed results are not presented here, a cyclic loading test was conducted up to 10 cycles using a different sample, and a similar observation was obtained. This indicates that, within the number of cycles tested in this study, the number of cycles had little effect on the strain distribution. It should be noted that the strains at the upper and lower ends of the gauge section tended to become more pronounced as the number of cycles increased. This would result from the non-uniform deformation due to the constraint of the clamping.

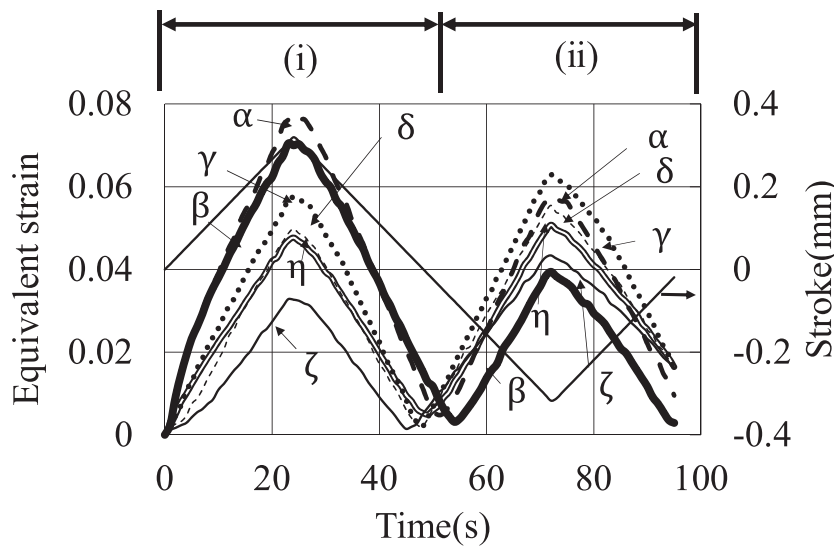

Fig. 12. Evolution of local equivalent strains as a function of time measured at points $\alpha$ to $\eta$ shown in Fig. 11.

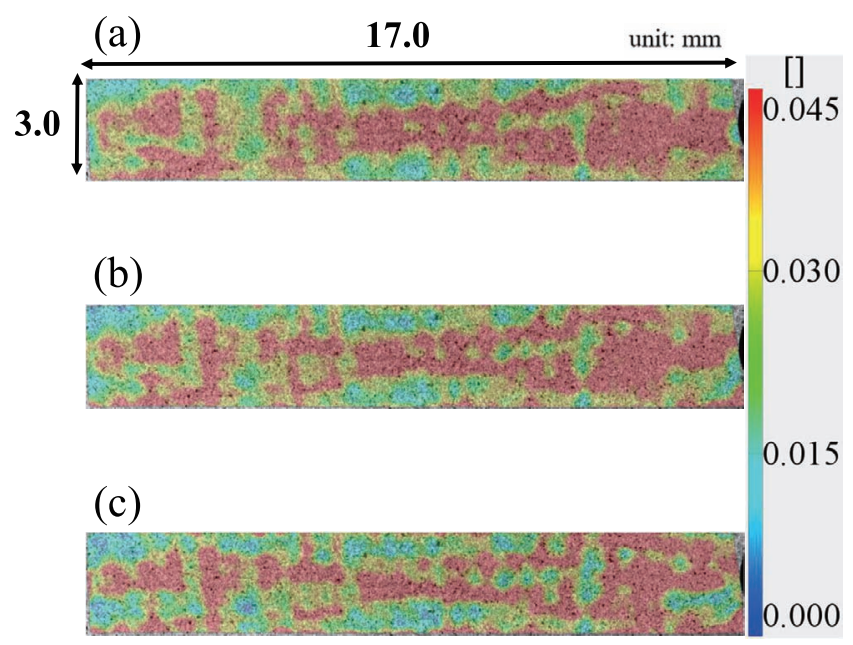

Fig. 13. Equivalent strain distributions measured at points (a) c, (b) c', and (c) c" shown in Fig. 10. (Online version in color.)

\subsubsection{Relationship between Strain Distribution and Grain Boundaries}

It is well-known that strain localization is affected strongly by grain boundaries. Therefore, the relationship between strain localization and grain boundaries was investigated. The grain boundaries of the specimen were extracted manually from photographs of the gauge section of the etched specimen. Although detail results are not provided here, it was confirmed that the manually extracted grain boundaries were in good agreements with grain boundaries measured using EBSD for some specific areas.

Figures 14(a), 14(b), 14(c) and 14(d) show the equivalent strain distributions measured at the points R, S, T, and $\mathrm{U}$ in Fig. 10, respectively, along with the extracted grain boundaries. In the present work, the displacement gradient in the areas of 9 subsets were calculated to evaluate strains. Because the subsets were arranged uniformly in the domain, grain boundaries should cross these areas. Therefore, the assumption of the smoothness of displacement gradient within the area of 9 subsets was not necessarily guaranteed in the vicinity of grain boundaries, which would result in inaccurate strain calculations. However, our preliminary study showed that the strain distributions were quite smooth at any points on the measured area. This result indicates that 

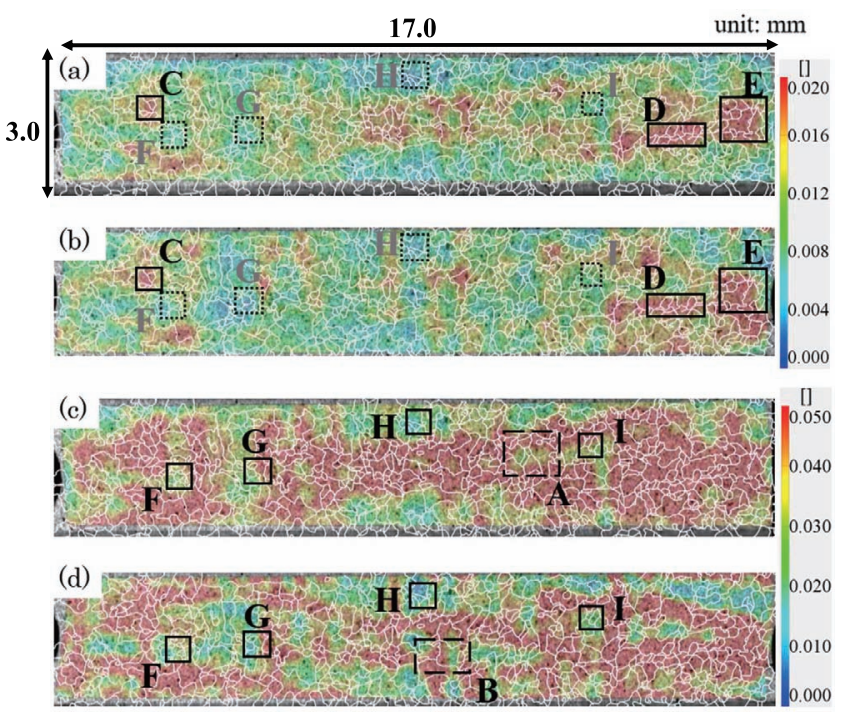

Fig. 14. Equivalent strain distributions measured at points (a) R, (b) S, (c) T and (d) U shown in Fig. 10. Grain boundaries are superimposed. (Online version in color.)

the effect of grain boundaries on the calculation of strains was negligible in the present work.

The points $R$ and $T$ were during the positive shear, whereas the points $\mathrm{S}$ and $\mathrm{U}$ were during the negative shear. Many of the boundaries of the strain localized areas were found to be along the grain boundaries during both the positive and negative shear. In contrast, the boundaries of the strain localized areas, i.e., significant strain gradients, appeared also within grains in some regions. For instance, Fig. 15(a) shows the enlarged figures of the regions A and B designated by the squares in Figs. 14(c) and 14(d). The boundaries of strain localized areas appeared within grains.

The heterogeneous strain distributions within grains observed in the regions A and B are investigated in detail. The grains highlighted with the black solid lines in Fig. 15(a) are focused on. The equivalent strain evolution at the four points $\mathrm{A} 1, \mathrm{~A} 2, \mathrm{~B} 1$, and $\mathrm{B} 2$ during the first cycle is shown in Fig. 15(b). The points A1 and B1 were selected from the strain localized regions, while the points $\mathrm{A} 2$ and B2 were from the regions with small strains. The equivalent strains evolved almost linearly irrespective of the points, similar to the results shown in Fig. 12. This result suggests that the heterogeneity of strain within the grains occurred also from the beginning of the deformation, consistent with the global heterogeneous strain distributions shown in Figs. 6 and 11.

It is also understood that grain boundary triple junctions are also significant for strain localization. Zhang et al. ${ }^{12}$ ) reported from their high-resolution DIC measurements of Ti-6Al-4V in tension that significant strain concentrations were observed at grain boundary triple junctions. Zhang and Tong $^{25)}$ observed dense slip-plane traces at a grain boundary triple junction in $\mathrm{Al}-0.5 \% \mathrm{Mg}$ polycrystal under tension. Sachtleber et al. ${ }^{2)}$ observed onset of strain localization at grain boundary triple junction, as also described in the introduction. Moreover, Tabata $e t$ al. $^{21)}$ also showed that plastic strains tended to be more concentrated at grain boundary triple and quadruple junctions in $\mathrm{Fe}-\mathrm{Si}-\mathrm{Mn}$ alloy under tension. Similar tendencies were also observed in Fig. 14.
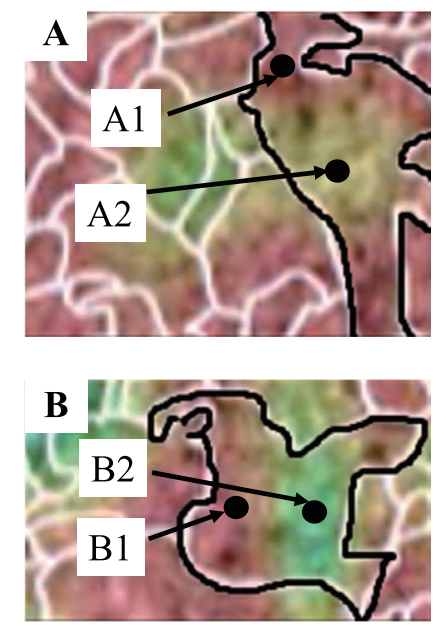

(a)

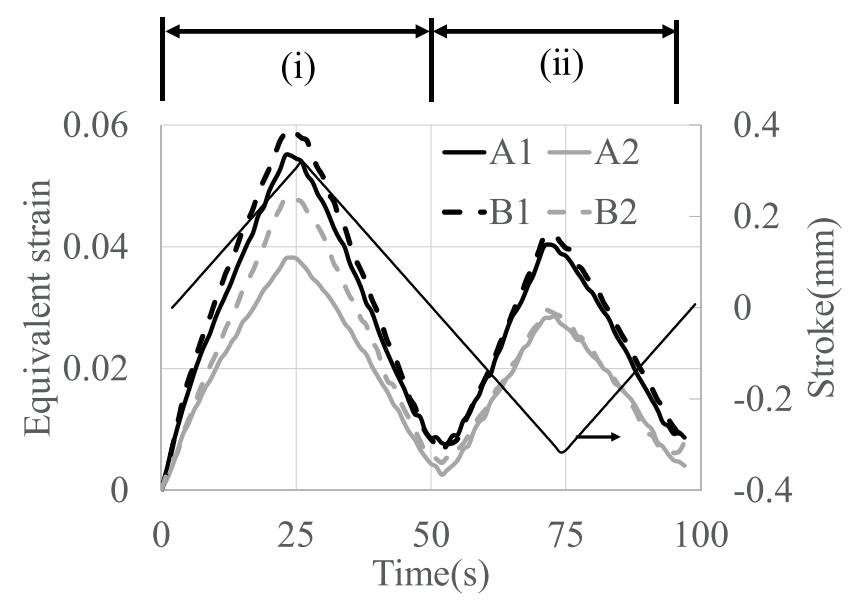

(b)

Fig. 15. Detailed distribution and evolution in the regions $\mathrm{A}$ and B shown in Figs. 14(c) and 14(d). (a) Enlarged figures of the regions $\mathrm{A}$ and $\mathrm{B}$, and (b) evolution of equivalent strains as a function of time measured at points A1, A2, B1 and B2 shown in (a). (Online version in color.)

For instance, the enlarged figures of the regions C, D, and E designated by the squares in Fig. 14(b) are shown in Fig. 16. Apparently, strong strain localization appeared in the vicinity of grain boundary triple junctions, as designated by the arrows. Moreover, the strain localization in the vicinity of grain boundary triple junctions tended to become parts of strain bands in the subsequent deformation, consistent with the previous studies.

However, it should be noted that strain bands do not always involve grain boundary triple junctions with strain localization, suggesting that the strain localization in the vicinity of grain boundary triple junctions is just one of several factors that induce strain bands.

Moreover, there were also a lot of grain boundary triple junctions which did not involve strain localization. For instance, in the regions $\mathrm{F}, \mathrm{G}, \mathrm{H}$, and I designated by the squares in Figs. 14(c) and 14(d), localized strains did not appear in the vicinity of triple junctions during both the positive and negative shear. The mechanism that yields the difference between the grain boundary triple junctions with and without strain localization is still open to discussion ${ }^{21)}$ and will be studied in future works. 
(a)

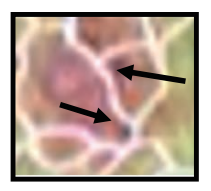

(b)

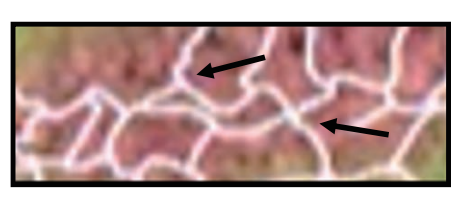

(c)

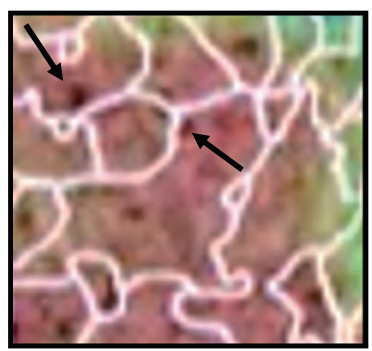

Fig. 16. Enlarged figures of the regions (a) C, (b) D and (c) E shown in Fig. 14(b). (Online version in color.)

As mentioned above, because differences in crystal orientations between grains should affect significantly heterogeneous strain distributions, it is necessary to consider not only grain boundaries but also crystal orientations. The relationships between crystal orientations, microscopic strain fields, and macroscopic strain fields are being examined experimentally and numerically. Their results will be reported elsewhere.

\section{Conclusions}

In this study, evolution of strain distributions at the grain level in an ultralow carbon steel with coarse grains were investigated under uniaxial tensile and cyclic simple shear loadings by using a digital image correlation method. The conclusions obtained are as follows.

(1) Localized strain bands are observed in both uniaxial tensile and simple shear deformations. The patterns of heterogeneous strain distribution are determined at the beginning of the deformation. However, local strain evolution is almost linear. It is presumed that the directions of the strain bands are strongly correlated with the planes of macroscopic maximum and minimum shear stresses.

(2) The evolution of strain bands is almost independent of the sheet thickness. Moreover, clear relationship between strain and surface roughness distributions is not observed. These results indicate that through-thickness deformation has little effect on in-plane non-uniform strain distributions.

(3) The results of cyclic simple shear test show that strain distributions are different depending on the load- ing direction, suggesting that the strain heterogeneity and the onset of strain bands have directional dependences. In contrast, the number of cycles has little effect on strain distributions.

(4) Localized strains tend to be formed along grain boundaries, but sometimes boundaries of strain bands also occur within grains. The strain heterogeneity within grains appears also at the beginning of the deformation.

\section{Acknowledgements}

The authors would like to thank Mr. Sohei Uchida of the Osaka Prefectural Institute of Technology for his cooperation in EBSD measurement. This work was partly supported by JSPS KAKENHI Grant number 20H02480 and Amada Foundation Grant number AF-2019004-A3.

\section{REFERENCES}

1) D. Raabe, M. Sachtleber, Z. Zhao, F. Roters and S. Zaefferer: Acta Mater., 49 (2001), 3433.

2) M. Sachtleber, Z. Zhao and D. Raabe: Mater. Sci. Eng. A, 336 (2002), 81 .

3) N. Nakada, K.-I. Ikeda, H. Shuto, T. Yokoi, T. Tsuchiyama, S. Hata, H. Nakashima and S. Takaki: ISIJ Int., 56 (2016), 2077.

4) K. Hasegawa, K. Kawamura, T. Urabe and Y. Hosoya: ISIJ Int., 44 (2004), 603.

5) B. M. Schroeter and D. L. McDowell: Int. J. Plast., 19 (2003), 1355.

6) E. Héripré, M. Dexet, J. Crépin, L. Gélébart, A. Roos, M. Bornert and D. Caldemaison: Int. J. Plast., 23 (2007), 1512.

7) C. Efstathiou, H. Sehitoglu and J. Lambros: Int. J. Plast., 26 (2010), 93.

8) W. Z. Abuzaid, M. D. Sangid, J. D. Carroll, H. Sehitoglu and J. Lambros: J. Mech. Phys. Solids, 60 (2012), 1201.

9) Y. Guo, F. Li, T. Suo, Z. Tang and Y. Li: Mech. Mater., 62 (2013), 80 .

10) A. Guery, F. Hild, F. Latourte and S. Roux: Int. J. Plast., 81 (2016), 249.

11) D. Lunt, J. Quinta da Fonseca, D. Rugg and M. Preuss: Mater. Sci. Eng. A, 680 (2017), 444.

12) Z. Zhang, D. Lunt, H. Abdolvand, A. J. Wilkinson, M. Preuss and F. P. E. Dunne: Int. J. Plast., 108 (2018), 88.

13) R. Kakimoto, M. Koyama and K. Tsuzaki: Tetsu-to-Hagané, 105 (2019), 222 (in Japanese).

14) J. H. Liu, N. Vanderesse, J.-C. Stinville, T. M. Pollock, P. Bocher and D. Texier: Acta Mater., 169 (2019), 260.

15) N. Nakada, M. Nishiyama, N. Koga, T. Tsuchiyama and S. Takaki: Tetsu-to-Hagané, 100 (2014), 1238 (in Japanese).

16) N. Ishikawa, K. Yasuda, H. Sueyoshi, S. Endo, H. Ikeda, T. Morikawa and K. Higashida: Acta Mater., 97 (2015), 257.

17) M. Fujisawa, R. Mauchi, T. Morikawa, M. Tanaka and K. Higashida: Tetsu-to-Hagané, 102 (2016), 405 (in Japanese).

18) N. Koga, M. Suzuki and O. Umezawa: Procedia Manuf., 15 (2018), 1656.

19) N. Koga, T. Yamashita and O. Umezawa: ISIJ Int., 60 (2020), 2083.

20) N. Koga, T. Nameki, O. Umezawa, V. Tschan and K.-P. Weiss: Mater. Sci. Eng. A, 801 (2021), 140442.

21) R. Tabata, H. Tsuruzono, S. Sadamatsu and Y. Adachi: Netsu Shori (J. Jpn. Soc. Heat Treat.), 55 (2015), 368 (in Japanese).

22) A. Venkataraman, M. Linne, S. Daly and M. D. Sangid: Materialia, 8 (2019), 100499.

23) F. Delaire, J. L. Raphanel and C. Rey: Acta Mater., 48 (2000), 1075.

24) T. Hoc, J. Crépin, L. Gélébart and A. Zaoui: Acta Mater., 51 (2003), 5477.

25) N. Zhang and W. Tong: Int. J. Plast., 20 (2004), 523.

26) Z. Zhao, M. Ramesh, D. Raabe, A. M. Cuitiño and R. Radovitzky: Int. J. Plast., 24 (2008), 2278.

27) H. Lim, J. D. Carroll, C. C. Battaile, T. E. Buchheit, B. Boyce and C. Weinberger: Int. J. Plast., 60 (2014), 1.

28) P. Baudoin, T. Hama and H. Takuda: Int. J. Plast., 115 (2019), 111.

29) N. Grilli, P. Earp, A. C. F. Cocks, J. Marrow and E. Tarleton: J. Mech. Phys. Solids, 135 (2020), 103800.

30) S. Bouvier, H. Haddadi, P. Levée and C. Teodosiu: J. Mater. Process. Technol., 172 (2006), 96. 